\title{
CONTROL OVER PERFORMANCE OF QUBIT-BASED SENSORS
}

\author{
Sergey Borisenok \\ Department of Electrical and Electronics Engineering \\ Faculty of Engineering \\ Abdullah Gül University \\ Turkey \\ sergey.borisenok@agu.edu.tr \\ Feza Gürsey Center for Physics and Mathematics \\ Boğaziçi University \\ Turkey \\ borisenok@gmail.com
}

\section{Article history:}

Received 28.09.2018, Accepted 15.11.2018

\begin{abstract}
The extreme sensitivity of quantum systems towards the external perturbations and in the same time their ability to be strongly coupled to the measured target field makes them to be stable under the environmental noise. A high quality quantum sensor can be engineered even on the platform of a single trapped qubit There is a variety of optimal and sub-optimal algorithms for effective control over the quantum system states. Here we discuss the opportunity to improve the efficiency of the external field quantum sensor based on a single qubit via its feedback tracking.
\end{abstract}

\section{Key words}

Quantum sensors, feedback tracking algorithms, quantum Fisher information.

\section{Introduction}

In the years 2017-2018 we observe a distinct progress in different engineering applications of quantum systems trapped by the specific shapes of potentials and interacting with static impurities. Such systems are used for the purpose of quantum computation, quantum simulations of many-body physics and for development of precise quantum analyzers and sensors.

The extreme sensitivity of quantum systems towards the external perturbations and in the same time their ability to be strongly coupled to the measured target field makes them to be stable under the environmental noise. For example, it is the noise cancellation by the coherent backaction for quantum dot-base sensors has been developed in [Hell, Wegewijs, and DiVincenzo, 2014; 2016]. The measurement backaction induced by the noise of electron cotunneling through the sensor is mitigated by the recently identified coherent backaction [Hell, Wegewijs, and DiVincenzo, 2014] arising from quantum fluctuations. The main idea of the approach is that for this is that the sensor itself is also a microscopic system, so that the action of the measured object on the sensor dynamics is not negligible, which then in turn affects the backaction of the sensor on the measured part. This coupling should be considered in the corresponding terms in the total Hamiltonian for the quantum dynamics equations describing the measurement action, and they represent a backaction of the measurement on the measured object [Hell, Wegewijs, and DiVincenzo, 2014]. The coherent backaction is an integral part of the total backaction together with stochastic and dissipative backaction, leading to the cancellation of cotunneling noise [Hell, Wegewijs, and DiVincenzo, 2016]. The effect of back-actions on dynamics of the system is taken into account by calculating the self-energies and the non-equilibrium Green's functions [Tabatabaei, 2017].

Thus, a high quality quantum sensor can be engineered even on the platform of a single qubit. Some approaches developed for quantum dot-based sensors could be also useful for the algorithmic concepts of trapped qubit-based sensors.

In this paper we discuss a feedback algorithm for the control over the performance of a single qubit-based sensor. The target function of the control is expressed 
via the quantum analog of the Fisher parameter. It is driven by the Kolesnikov's "synergetic" approach towards the target attractor maximizing the Fisher parameter, i.e. minimazing the lower bound of the uncertainty for the sensor measurement procedure.

\section{Qubit under the External Control Field}

Let's consider a qubit, two-level quantum system, attached to a quantum bath [Pechen and Borisenok, 2015]. The Lindblad dynamical equation for density operator is given by:

$$
\begin{aligned}
& \frac{d \rho}{d t}=-i\left[H_{0}+u(t) V, \rho\right]+ \\
& +\gamma\left[(n(t)+1) L_{12}(\rho)+n(t) L_{21}(\rho)\right]
\end{aligned}
$$

with Einstein coefficient $\gamma>1$ for the transition between two states 1 ("the ground level") and 2 ('the excited level'); and the Lindblad operators:

$L_{12}=2 \rho_{22} P_{1}-P_{2} \rho-\rho P_{2} ; L_{21}=2 \rho_{11} P_{2}-P_{1} \rho-\rho P_{1}$.

In case if the decay is absent or weak, the system preserves its evolution on the surface of the Bloch sphere $x^{2}(t)+y^{2}(t)+z^{2}(t)=1$ with the dynamical system in the dimensionless form [Borisenok, Fradkov, and Proskurnikov, 2010]:

$$
\dot{x}=u \cdot z ; \dot{y}=z ; \dot{z}=-y-u \cdot x,
$$

where

$$
\begin{aligned}
& x=\rho_{22}-\rho_{11} ; \\
& y=\rho_{12} \exp \{i \omega t\}+\rho_{21} \exp \{-i \omega t\} \\
& z=i\left[\rho_{12} \exp \{i \omega t\}-\rho_{21} \exp \{-i \omega t\}\right] .
\end{aligned}
$$

The energy distance between two level is given by $\omega=$ $\left(E_{2}-E_{1}\right) / \hbar$.

\section{Control over the Sensor Performance}

The performance of the quantum sensor measurement is based on the quantum analog [Braunstein and Caves, 1994; Refregier, 2012] of the classical Cramer-Rao lower bound (CRLB) theorem [Cramer, 1946; Rao, 1945]. CRLB defines a lower bound of uncertainty of the inferred value $\Delta u_{m}$ of the measured parameter $u_{m}$ [Refregier, 2012]:

$$
\left(\Delta u_{m}\right)^{2} \geq \frac{1}{N F}
$$

Here $N$ stands for the numbers of measuring elements (qubits in our case). The parameter $F$ is the quantum analog of classical Fisher information, for the
Jachymski-Wasak-Idziaszek (JWI) measurement protocol and other similar types of sensors it is given by [Jachymski, Wasak, and Idziaszek, 2018]:

$$
F=\sum_{s} \frac{1}{P\left(s \mid u_{m}\right)}\left(\frac{\partial P\left(s \mid u_{m}\right)}{\partial u_{m}}\right)^{2}
$$

where the summation is made over the full set $\{s\}$ of quantum states, and $P\left(s \mid u_{m}\right)$ are the probabilities to get the corresponding state $s$ under the action of the external measured dimensionless field $u_{m}$. Thus, the maximization of the Fisher parameter $F$ minimizes the lower bound of the uncertainty (3).

\subsection{Fisher Parameter for One Sensoring Qubit}

For the quantum system (2) making the evolution over the surface of the Bloch sphere without decay $\rho_{11}+$ $\rho_{22}=1$. By that its inversion $x=\rho_{22}-\rho_{11}$ defines the qubit state: $\rho_{11}=(1-x) / 2$ and $\rho_{22}=(1+x) / 2$. That implies for (4):

$$
F=\frac{1}{\rho_{11}}\left(\frac{\partial \rho_{11}}{\partial u}\right)^{2}+\frac{1}{\rho_{22}}\left(\frac{\partial \rho_{22}}{\partial u}\right)^{2}
$$

We remind that dimensionless $u(t)$ stands here for the external field(s). Under the substitution of the density matrix elements via the inversion and considering the dynamical system (2) Eq. (5) becomes:

$$
F=\frac{1}{1-x^{2}}\left(\frac{\partial x}{\partial u}\right)^{2}
$$

One can see that the Fisher parameter $F$ becomes infinite under two natural limits $x= \pm 1$, which correspond to two pure states of the qubit (the pure ground and the pure exited states), where the uncertainty, sure, is zero by (3).

\section{2 "Target Attractor" Algorithm for the Control over the Sensor Performance}

Let's apply now Kolesnikov's target attractor algorithm with a positive constant $T$ [Kolesnikov, 2012]:

$$
\dot{\psi}=-\frac{\psi}{T}
$$

to track the control goal to zero:

$$
\psi=\left(\frac{\partial u}{\partial x}\right)^{2} \rightarrow 0
$$

By the simplification we get:

$$
\frac{\partial}{\partial x}\left[\frac{d u}{d t}+\frac{1}{2 T} \cdot u\right]=0
$$


Let's consider two components of the external field: $u=u_{m}+u_{c}$, where $u_{m}$ is a measured part and $u_{c}$ is a control signal. Then the tracking algorithm is given by:

$$
\frac{d u_{c}}{d t}+\frac{u_{c}}{2 T}=-\frac{d u_{m}}{d t}-\frac{u_{m}}{2 T}
$$

with the solution:

$$
u_{c}(t)=e^{-t / 2 T}\left[u_{c}(0)-\int_{0}^{t} e^{\tau / 2 T} f\left(u_{m}(\tau)\right) d \tau\right]
$$

with the given function of the measured field:

$$
f\left(u_{m}(\tau)\right)=\frac{d u_{m}(\tau)}{d \tau}+\frac{u_{m}(\tau)}{2 T}
$$

The TA feedback is tracking the measured field $u_{m}(t)$ as a dynamical function and drive the additional control field $u_{c}(t)$ to minimize the uncertainty (3). If the function $u_{m}(t)$ and its first time derivative are finite (virtually any real physical measured field satisfies to such criteria), the algorithm (11) converges.

This approach can be easily extended for the bigger number of the measuring qubits.

\section{Conclusion}

We feedback target attractor control is able to provide the efficient control over the performance of single- and few-qubit based quantum sensors. The control field $u_{c}$ is restored via the measured external field $u_{m}$.

Our algorithmic approach could be realized experimentally in a set of physical systems, like nitrogen vacancy centers in diamonds, ultracold atoms in magnetic fields and others.

\section{Acknowledgements}

The work was performed in Institute of Problems in Mechanical Engineering of Russian Academy of
Sciences (IPME RAS), supported by Russian Science Foundation (grant 14-29-00142).

\section{References}

Borisenok, S., Fradkov, A., and Proskurnikov, A. (2010). Speed gradient control of qubit state, Periodic Control Systems, 4(1), pp. 81-86.

Braunstein, S. L. and Caves, C. M. (1994). Statistical distance and the geometry of quantum states, Phys. Rev. Lett., 72, p. 3439.

Cramer, H. (1946). Mathematical Methods of Statistics. Princeton Univ. Press. Princeton, NJ.

Hell, M., Wegewijs, M.R., and DiVincenzo, D.P. (2014). Coherent backaction of quantum dot detectors: Qubit isospin precession, Phys. Rev. B, 89, p. 195405.

Hell, M., Wegewijs, M.R., and DiVincenzo, D.P. (2014). Qubit quantum-dot sensors: Noise cancellation by coherent backaction, initial slips, and elliptical precession, Phys. Rev. B, 93, p. 045418.

Jachymski, K., Wasak, T., and Idziaszek, Z. (2018). Single-atom transistor as a precise magnetic field sensor. Phys. Rev. Lett., 120, p. 013401

Kolesnikov, A. (2012). Synergetic Control Methods for Complex Systems. URSS Publ. Moscow.

Pechen, A.N. and Borisenok, S. (2015). Energy transfer in two-level quantum wystems via speed gradient-based algorithm. IFAC-PapersOnLine, 48(11), pp. 446-450.

Rao, C. R. (1945). Information and the accuracy attainable in the estimation of statistical parameters, Bull. Calcutta Math. Soc., 37, pp. 81-89.

Refregier, P. (2012). Noise theory and application to physics: from fluctuations to information. Springer Science \& Business Media. New York.

Tabatabaei, S.M. (2017). Perturbative approach to the capacitive interaction between a sensor quantum dot and a charge qubit, Phys. Rev. B, 95, p. 155113. 